\title{
Relativistic Propagation of Linearly/Circularly Polarized Laser Radiation in Plasmas
}

\author{
Sonu Sen, ${ }^{1}$ Meenu Asthana Varshney, ${ }^{2}$ and Dinesh Varshney ${ }^{3}$ \\ ${ }^{1}$ Department of Engineering Physics, Indore Institute of Science and Technology, Indore 453331, India \\ ${ }^{2}$ Department of Physics, M. B. Khalsa College, Indore 452002, India \\ ${ }^{3}$ School of Physics, Vigyan Bhawan, Devi Ahilya University, Khandwa Road Campus, Indore 452001, India \\ Correspondence should be addressed to Sonu Sen; ssen.plasma@gmail.com
}

Received 27 May 2013; Accepted 25 July 2013

Academic Editors: M. Hu, V. Matejec, and J. Shibayama

Copyright (C) 2013 Sonu Sen et al. This is an open access article distributed under the Creative Commons Attribution License, which permits unrestricted use, distribution, and reproduction in any medium, provided the original work is properly cited.

Paraxial theory of relativistic self-focusing of Gaussian laser beams in plasmas for arbitrary magnitude of intensity of the beam has been presented in this paper. The nonlinearity in the dielectric constant arises on account of relativistic variation of mass. An appropriate expression for the nonlinear dielectric constant has been used to study laser beam propagation for linearly/circularly polarized wave. The variation of beamwidth parameter with distance of propagation, self-trapping condition, and critical power has been evaluated. The saturating nature of nonlinearity yields two values of critical power of the beam $\left(P_{\mathrm{cr} 1}\right.$ and $\left.P_{\mathrm{cr} 2}\right)$ for self-focusing. When $P<P_{\mathrm{cr} 1}<P_{\mathrm{cr} 2}$ the beam diverges. When $P_{\mathrm{cr} 1}<P<P_{\mathrm{cr} 2}$ the beam first converges then diverges and so on. When $P>P_{\mathrm{cr} 2}$ the beam first diverges and then converges and so on. Numerical estimates are made for linearly/circularly polarized wave applicable for typical values of relativistic laser-plasma interaction process in underdense and overdense plasmas. Since the relativistic mechanism is instantaneous, this theory is applicable to understanding of self-focusing of laser pulses.

\section{Introduction}

The interaction of ultrahigh-power laser beams with plasmas is not only of technological importance but also rich in a variety of nonlinear phenomena. These phenomena become particularly interesting and involved when the laser power is sufficiently intense to cause the electron oscillation (quiver) velocity to become relativistic [1]. An important process that can affect the size of the focused spot of the radiation is selffocusing. In recent years the field of laser plasma interaction in the relativistic regime has been identified as an emerging area and is often referred to as high-field science. Several complex phenomena are included in the area of high-field science. These phenomena (on account of their nonlinear nature) are all significantly affected by the field distribution in the beam, and hence, self-focusing occupies a unique position in the field as it affects all other phenomena.

Relativistic electron motion in a plasma due to an intense laser pulse modifies the refractive index and leads to two effects: relativistic induced transparency and relativistic selffocusing. In dense plasma with $\omega<\omega_{p}$, light cannot propagate and is reflected from the surface. However, for relativistic intensities generating large $\gamma$ factors, the plasma becomes transparent. The dependence of the electron mass on intensity causes significant change of characteristic properties of the plasma and its nonlinear processes; the increase in effective electron mass decreases the electron plasma frequency and makes the penetration of electromagnetic waves into the overdense area of plasma, which is known as self-induced transparency or electromagnetically induced transparency, and is the cause of relativistic self-focusing. A combination of the relativistic self-focusing and relativistic induced transparency enables transmission of laser energy deep into plasmas which is useful for fast ignition of inertial fusion [2]. This so-called penetration sensitively depends on the focal position of the laser intensity due to the inhomogeneous density profile of the plasma and convergence of the laser pulse by final focusing optics.

For ultrafast laser pulses lasting the order of a picosecond or less, the drift velocity of electrons in a plasma can be comparable to the velocity of light, causing a significant increase in the mass of the electron and consequently in the 
effective dielectric constant of the plasma. This leads to selffocusing of the laser beam, as pointed out [3]. The fact that the relativistic mechanism is the only mechanism of selffocusing that can manifest itself for subpicosecond pulses makes the understanding of this mechanism very important $[4,5]$. The earlier analysis concerned with relativistic selffocusing of laser beams in plasmas ignored the effect of the saturating nature of the nonlinear dielectric constant and was essentially a perturbation treatment, based on the quadratic dependence of the dielectric constant on the electric field of the beam [6]. Hence, such an analysis has limited applicability to understand the self-focusing of beams with arbitrarily large electric field and consequently arbitrarily large nonlinear part of the dielectric constant.

Relativistic focusing of radiation beams in plasma is applicable to pulse length $\tau_{R}$ in the range $1 / \omega<\tau_{R}<r_{s} / C_{s}$, where $C_{s}$ is the ion-acoustic speed [7], and can arise owing to the creation of a density depression in the plasma as well as to an increase in the electron mass due to relativistic effects. The time scale for self-focusing due to a density depression is longer than that for self-focusing due to relativistic mass increase. The latter time scale is $\tau_{R} \approx \omega^{-1}$, where $\omega$ is the radiation frequency and is assumed to be much greater than the electron plasma frequency. The analysis presented here will be concerned only with relativistic self-focusing on a time scale sufficiently short that the plasma density profile does not evolve significantly under the influence of the radiation beam. This implies that the pulse length $\tau_{R}$ of the radiation beam must be short compared with $\tau_{s}=r_{s} / C_{s}$ (the time scale for the density depression to occur) and, of course, long compared with a radiation period $\tau_{R}$. We make use of the steady-state paraxial-ray theory, as developed by Akhamanov et al. [8] and Sodha et al. [9].

The organization of the paper is as follows. In Section 2 the nonlinear dielectric constant due to relativistic variation of mass for a time-harmonic plane wave (i.e., a linearly/circularly polarized wave) is found. Section 3 is concerned with the relativistic self-focusing equation relating the variation of beamwidth parameter with distance of propagation. The self-trapping condition and the critical power are evaluated in Section 4. Results and discussion are made in Section 5 .

\section{Nonlinear Dielectric Constant of the Plasma}

The relativistic equation of motion for a point charge is

$$
\frac{d}{d t}\left[\frac{m \mathbf{v}}{\left(1-v^{2} / c^{2}\right)^{1 / 2}}\right]=e[\mathbf{E}+(\mathbf{v} \times \mathbf{B})],
$$

where $m$ is the rest mass of a point charge, $c$ is the velocity of light in vacuum, $\mathbf{E}$ is the electric field, and $\mathbf{B}$ is the wave magnetic field. For a homogeneous plane wave traveling in the direction of a unit vector $\mathbf{n}$, the electric and magnetic vectors are perpendicular to $\mathbf{n}$ and are related by

$$
\mathbf{B}=\mathbf{n} \times\left(\frac{\mathbf{E}}{c}\right) .
$$

Substituting for $\mathbf{B}$ on the right-hand side of the equation of motion, (1) gives

$$
\frac{d}{d t}\left[\frac{\mathbf{v}}{\left(1-v^{2} / c^{2}\right)^{1 / 2}}\right]=\frac{e}{m}\left(1-\frac{\mathbf{n} \cdot \mathbf{v}}{c}\right) \mathbf{E}+\frac{e}{m c}(\mathbf{E} \cdot \mathbf{v}) \mathbf{n} .
$$

Considering the scalar product of (3) with $\mathbf{n}$ yields

$$
\frac{d}{d t}\left[\frac{\mathbf{v}}{\left(1-v^{2} / c^{2}\right)^{1 / 2}}\right]=\frac{e}{m c}(\mathbf{E} \cdot \mathbf{v}) .
$$

Equation (4) together with the energy equation

$$
\frac{d}{d t}\left[\frac{m c^{2}}{\left(1-v^{2} / c^{2}\right)^{1 / 2}}\right]=e \mathbf{E} \cdot \mathbf{v}
$$

establishing that

$$
\lambda \equiv\left[\frac{1-\mathbf{n} \cdot \mathbf{v} / c}{\left(1-v^{2} / c^{2}\right)^{1 / 2}}\right]
$$

is constant. We now eliminate $t$ in favour of the phase variable

$$
\varphi=\omega\left(t-\frac{\mathbf{n} \cdot \mathbf{r}}{c}\right) .
$$

Here $\omega$ is the angular frequency of the wave, and $\mathbf{r}$ is the positive vector of the particle. Then

$$
\mathbf{v}=\frac{d \mathbf{r}}{d t}=\omega\left(1-\frac{\mathbf{n} \cdot \mathbf{v}}{c}\right) \mathbf{r}^{\prime},
$$

where the prime denotes differentiation with respect to $\varphi$, and using (6)

$$
\begin{aligned}
\frac{d}{d t}\left[\frac{\mathbf{v}}{\left(1-v^{2} / c^{2}\right)^{1 / 2}}\right] & =\lambda \frac{d}{d t}\left(\frac{\mathbf{v}}{1-\mathbf{n} \cdot \mathbf{v} / c}\right) \\
& =\omega^{2} \lambda\left(1-\frac{\mathbf{n} \cdot \mathbf{v}}{c}\right) \mathbf{r}^{\prime \prime} .
\end{aligned}
$$

Substitution of (8) and (9) into (3) enables the factor (1 - $\mathbf{n}$. $\mathbf{v} / c)$ to be cleared and leaves

$$
\mathbf{r}^{\prime \prime}=\frac{e}{\lambda m \omega^{2}}\left[\mathbf{E}+\frac{\omega}{c}\left(\mathbf{E} \cdot \mathbf{r}^{\prime}\right) \mathbf{n}\right]
$$

which is a second-order linear differential equation for $\mathbf{r}$ as a function of $\varphi$. The dielectric constant of the plasma is given by

$$
\varepsilon=1-\left(\frac{\omega_{p}^{2}}{\omega^{2}}\right)\left(\frac{m}{m_{r}}\right)
$$

where $m_{r}=\gamma \cdot m$ is the relativistic mass, $\gamma=\left(1-v^{2} / c^{2}\right)^{1 / 2}$ is the relativistic factor, and $\omega_{p}=\left(4 \pi n_{0} e^{2} / m\right)^{1 / 2}$ is the plasma 
frequency in the absence of the beam. For a circularly polarized wave, the components of (10) are

$$
\begin{aligned}
x^{\prime \prime} & =\frac{e E \cos \varphi}{\lambda m \omega^{2}}, \\
y^{\prime \prime} & =\frac{-e E \sin \varphi}{\lambda m \omega^{2}}, \\
z^{\prime \prime} & =\frac{e E x^{\prime} \cos \varphi}{8 m^{2} \lambda^{2} c \omega^{3}},
\end{aligned}
$$

where $\varphi=(\omega t-k z)$ is the phase, and $\omega$ and $k$ approximately satisfy the linear dispersion relation

$$
\omega^{2}=\omega_{p}^{2}+c^{2} k^{2}
$$

Using (12), the average relativistic factor is

$$
\langle\gamma\rangle=\left[1+\frac{e^{2} E^{2}}{m^{2} \omega^{2} c^{2}} C_{1}\right]^{1 / 2},
$$

where

$$
C_{1}=1+\frac{e^{2} E^{2}}{16 \lambda^{2} m^{2} \omega^{2} c^{2}}
$$

for self-focusing considering the order of electric field up to second order and that the value of $C_{1}$ is unity for circularly polarized wave [10] and half for linearly polarized wave. The dielectric constant can be written as [9]

$$
\varepsilon=\varepsilon_{0}+\phi\left(E_{0} E_{0}^{*}\right)
$$

where

$$
\varepsilon_{0}=1-\left(\frac{\omega_{p}^{2}}{\omega^{2}}\right)
$$

is the linear part of dielectric constant,

$$
\phi\left(E_{0} E_{0}^{*}\right)=\frac{\omega_{p 0}^{2}}{\omega^{2}}\left[1+\left(1-\frac{1}{2} \alpha E_{0} E_{0}^{*} C_{1}\right)^{-1 / 2}\right]
$$

is the nonlinear relativistic term, and

$$
\alpha=\frac{2 e^{2}}{m^{2} \omega^{2} c^{2}} .
$$

The effective dielectric constant for arbitrary large nonlinearity can be written as

$$
\varepsilon=\varepsilon_{0}^{\prime}-\varepsilon_{1}(f) r^{2}
$$

where $f(z)$ is the dimensionless beamwidth parameter and $\varepsilon_{0}^{\prime}$ and $\varepsilon_{1}$ represent, respectively, the linear and nonlinear dielectric constant for arbitrary nonlinearity.

\section{Relativistic Self-Focusing Equation}

Consider the propagation of Gaussian laser beam of frequency $\omega$ along $z$-direction; at $z=0$ the intensity distribution of the beam is given by

$$
E E^{*}=E_{0}^{2} \exp \left(\frac{-r^{2}}{r_{0}^{2}}\right),
$$

and here, " $r$ " is the radial coordinate of the cylindrical coordinate system, " $r_{0}$ " is the initial beamwidth, and $E_{0}$ is the axial amplitude. The wave equation governing the electric vector of beam in plasmas with the effective dielectric constant $(20)$ is

$$
\nabla^{2} \mathbf{E}-\nabla(\nabla \cdot \mathbf{E})+\frac{\omega^{2}}{c^{2}} \varepsilon \mathbf{E}=0
$$

To solve the prevoius equation, the Wentzel-KramersBrillouin (WKB) approximation has been used. In the WKB approximation, the second term $\nabla(\nabla \cdot \mathbf{E})$ of (22) has been neglected, which is justified when $\left(c^{2} / \omega^{2}\right)\left|1 / \varepsilon \nabla^{2} \ln \varepsilon\right| \ll 1$. The electric vector of the main beam can be expressed as

$$
\nabla^{2} \mathbf{E}+\frac{\omega^{2}}{c^{2}} \varepsilon \mathbf{E}=0
$$

In the presence of the modified background electron concentration due to relativistic nonlinearity, the intensity distribution of the beam inside the plasma can be obtained by using the $\mathrm{WKB}$ and paraxial ray approximations. Following Akhmanov et al. [8] and Sodha et al. [9] the solution for $\mathbf{E}$ can be written as

$$
\mathbf{E}(r, z)=A(r, z)\left[\frac{k(0)}{k(f)}\right]^{1 / 2} \exp \left(-i \int k(f) d z\right)
$$

where

$$
\begin{gathered}
k(f)=\frac{\omega}{c}\left[\varepsilon_{0}^{\prime}(f)\right]^{1 / 2}, \\
k(0)=\frac{\omega}{c}\left[\varepsilon_{0}^{\prime}(f=1)\right]^{1 / 2} .
\end{gathered}
$$

Substituting for $\mathbf{E}$ and $\varepsilon$ from (24) and (20) in (23), one obtains

$$
-2 i k(f) \frac{\partial A}{\partial z}+\frac{\partial^{2} A}{\partial r^{2}}+\frac{1}{r} \frac{\partial A}{\partial r}-\frac{\omega^{2}}{c^{2}} \varepsilon_{1}(f) r^{2} A=0 .
$$

Putting $A=A_{0}(r, z) \exp [-i k S(r, Z)]$ in (26) and separating real and imaginary parts we get

$$
\begin{aligned}
2\left(\frac{\partial S}{\partial z}\right) & +\left(\frac{\partial S}{\partial r}\right)^{2}+\frac{\omega^{2}}{c^{2}} \frac{\varepsilon_{1}(f)}{k^{2}(f)} r^{2} \\
- & \frac{1}{k^{2}(f) A_{0}}\left(\frac{\partial^{2} A_{0}}{\partial r^{2}}+\frac{1}{r} \frac{\partial A_{0}}{\partial r}\right)=0 \\
\frac{\partial A_{0}^{2}}{\partial z^{2}}+\frac{\partial S}{\partial r} \frac{\partial A_{0}^{2}}{\partial r}+A_{0}^{2}\left(\frac{\partial^{2} S}{\partial r^{2}}+\frac{1}{r} \frac{\partial S}{\partial r}\right) & =0
\end{aligned}
$$


where $S(r, z)$ is called the eikonal and related to the curvature of the wavefront. For a slightly converging/diverging beam the solution for a Gaussian laser beam (21) can be written as

$$
\begin{aligned}
S & =\frac{r^{2}}{2} \beta(z)+\varphi(z), \\
A_{0}^{2} & =\frac{E_{0}^{2}}{f^{2}} \exp \left(\frac{-r^{2}}{r_{0}^{2} f^{2}}\right),
\end{aligned}
$$

where

$$
\beta=\frac{1}{f} \frac{d f}{d z} .
$$

It can be seen previously that $\beta$ is the inverse of radius of curvature of the wavefront and $r_{0} f$ is the width of the beam. In the geometrical optics approximation, $r=r_{0} f(z)$ represents a ray in a plane containing the $z$-axis. On substituting for $S$ into (27), using the paraxial ray approximation, such that $\left(r / r_{0} f\right) \ll 1$, equating the coefficients of $r^{2}$ on both sides of the resulting equations and substituting for $\beta$ into (29), the dimensionless beamwidth parameter is given by

$$
k^{2}(f) \frac{d^{2} f}{d z^{2}}=\frac{1}{r_{0}^{4} f^{3}}-\frac{\omega^{2}}{c^{2}} \varepsilon_{1}(f) f .
$$

Transforming the coordinate $z$ and the initial beamwidth $r_{0}$ to dimensionless forms

$$
\begin{gathered}
\xi=\left(\frac{z c}{r_{0}^{2} \omega}\right), \\
\rho_{0}=\left(\frac{r_{0} \omega}{c}\right),
\end{gathered}
$$

we get the characteristic beam propagation equation as

$$
\frac{d^{2} f}{d \xi^{2}}=\frac{1}{\varepsilon_{0}^{\prime}(f) f^{3}}-\frac{\rho_{0}^{2} r_{0}^{2} \varepsilon_{1}(f)}{\varepsilon_{0}^{\prime}(f)} f .
$$

Physically, (34) governs the variation of beamwidth parameter $f$ with distance of propagation. Substituting for $E$ from (24) and $S, A_{0}$, and $\beta$ from (29)-(31) into (20) and using the paraxial ray approximation, $\phi$ can be written as

$$
\begin{aligned}
& \phi\left(E E^{*}\right) \\
& \approx \phi\left(\frac{k(0)}{k(f)} \frac{E_{0}^{2}}{2 f^{2}}\right)-r^{2}\left\{\alpha \frac{\omega_{p 0}^{2}}{\omega^{2}} \frac{k(0)}{k(f)} \frac{E_{0}^{2}}{4 r_{0}^{2} f^{4}}\right. \\
& \times\left[1+\frac{k(0)}{k(f)} \alpha \frac{E_{0}^{2}}{2 f^{2}}\right. \\
& \left.\times\left(1+\frac{k(0)}{k(f)} \alpha \frac{E_{0}^{2}}{8 \lambda^{2} 2 f^{2}}\right)\right]^{-3 / 2} \\
& \left.\times\left[1+\frac{1}{4 \lambda^{2}} \frac{k(0)}{k(f)} \alpha \frac{E_{0}^{2}}{2 f^{2}}\right]\right\}
\end{aligned}
$$

correct to terms in $r^{2}$, where

$$
\begin{aligned}
& \phi\left(\frac{k(0)}{k(f)} \frac{E_{0}^{2}}{2 f^{2}}\right) \\
&=\frac{\omega_{p 0}^{2}}{\omega^{2}}\left\{1-\left[1+\frac{k(0)}{k(f)} \alpha \frac{E_{0}^{2}}{2 f^{2}}\right.\right. \\
&\left.\left.\times\left(1+\frac{k(0)}{k(f)} \alpha \frac{E_{0}^{2}}{16 \lambda^{2} 2 f^{2}}\right)\right]^{-1 / 2}\right\} .
\end{aligned}
$$

Equation (35) can put into the convenient form of (20), with [11]

$$
\begin{aligned}
& \varepsilon_{0}^{\prime}=\varepsilon_{0}+\frac{\omega_{p 0}^{2}}{\omega^{2}}\left\{1-\left[1+\frac{k(0)}{k(f)} \alpha \frac{E_{0}^{2}}{2 f^{2}}\right.\right.\left.\left.\times\left(1+\frac{k(0)}{k(f)} \alpha \frac{E_{0}^{2}}{16 \lambda^{2} 2 f^{2}}\right)\right]^{-1 / 2}\right\}, \\
& \varepsilon_{1}=\alpha \frac{\omega_{p 0}^{2}}{\omega^{2}} \frac{k(0)}{k(f)} \frac{E_{0}^{2}}{4 r_{0}^{2} f^{4}} \\
& \times\left\{\left[1+\frac{k(0)}{k(f)} \alpha \frac{E_{0}^{2}}{2 f^{2}}\left(1+\frac{k(0)}{k(f)} \alpha \frac{E_{0}^{2}}{8 \lambda^{2} 2 f^{2}}\right)\right]^{-3 / 2}\right. \\
&\left.\quad \times\left[1+\frac{1}{4 \lambda^{2}} \frac{k(0)}{k(f)} \alpha \frac{E_{0}^{2}}{2 f^{2}}\right]\right\} .
\end{aligned}
$$

Consider second order of nonlinearity, for circularly polarized wave; we can rewrite the previous set of equations as

$$
\begin{aligned}
& \varepsilon_{0}^{\prime}(f)=1-\frac{\omega_{p}^{2}}{\omega^{2}}\left(1+\frac{k(0)}{k(f)} \frac{\alpha E_{0}^{2}}{2 f^{2}}\right)^{-1 / 2}, \\
& \varepsilon_{1}(f)=\frac{\omega_{p}^{2}}{4 \omega^{2}} \frac{\alpha E_{0}^{2}}{r_{0}^{2} f^{4}}\left(1+\frac{k(0)}{k(f)} \frac{\alpha E_{0}^{2}}{2 f^{2}}\right)^{-3 / 2} .
\end{aligned}
$$

On substituting values of $\varepsilon_{0}^{\prime}(f)$ and $\varepsilon_{1}(f)$ from (38) in (34), the equation governing the beamwidth parameter is

$$
\begin{aligned}
\frac{d^{2} f}{d \xi^{2}}= & \frac{1}{\left[1-\frac{\omega_{p}^{2}}{\omega^{2}}\left(1+\frac{k(0)}{k(f)} \frac{\alpha E_{0}^{2}}{2 f^{2}}\right)^{-1 / 2}\right] f^{3}} \\
& -\frac{\frac{\omega_{p}^{2}}{4 \omega^{2}} \rho_{0}^{2} r_{0}^{2} \frac{\alpha E_{0}^{2}}{r_{0}^{2} f^{4}}\left(1+\frac{k(0)}{k(f)} \frac{\alpha E_{0}^{2}}{2 f^{2}}\right)^{-3 / 2}}{\left[1-\frac{\omega_{p}^{2}}{\omega^{2}}\left(1+\frac{k(0)}{k(f)} \frac{\alpha E_{0}^{2}}{2 f^{2}}\right)^{-1 / 2}\right]} .
\end{aligned}
$$




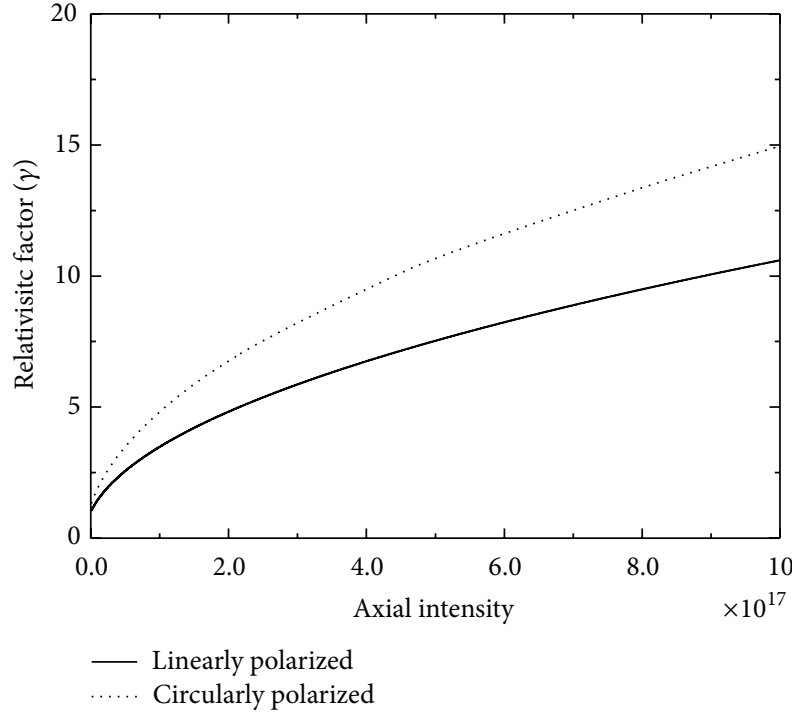

Figure 1: Dependence of relativistic factor on axial intensity for linearly and circularly polarized laser beam.

In (39) the first term on right-hand side is responsible for diffraction divergence of the beam, and the second term corresponds to convergence, arising due to nonlinearity through the relativistic factor of linearly or circularly polarized beam, which contributes to focusing.

\section{Self-Trapping Condition}

When the two terms on the right-hand side of (39) balance each other the beam propagates without convergence or divergence, which is referred to as uniform waveguide propagation or self-trapping of the beam. For an initial plane wavefront of the beam the initial conditions on $f$ are $f(\xi=$ $0)=1$ and $(d f / d \xi)_{\xi=0}=0$. As both the terms on right-hand side cancel each other hence at $z=0,\left(d^{2} f / d \xi^{2}\right)_{\xi=0}=0$; since $(d f / d \xi)$ is also zero and $f=1$ at $z=0, f=1$ for all values of $z$; in other words beam can propagate without convergence or divergence. Therefore, the condition for self-trapping is

$$
\rho_{0 \operatorname{Lin}}^{2}=\frac{4 \omega^{2}}{\omega_{p}^{2}} \frac{\left(1+\alpha E_{0}^{2} / 2\right)^{3 / 2}}{\alpha E_{0}^{2} / 2}
$$

for linearly polarized beam and

$$
\rho_{0 \mathrm{Cir}}^{2}=\frac{2 \omega^{2}}{\omega_{p}^{2}} \frac{\left(1+\alpha E_{0}^{2}\right)^{3 / 2}}{\alpha E_{0}^{2}}
$$

for circularly polarized beam. The critical power of the beam is

$$
\begin{gathered}
P=\frac{c}{8 \pi} \int_{0}^{\infty} \varepsilon^{1 / 2} E_{0 \mathrm{cr}}^{2} \exp \left(-\frac{r^{2}}{r_{0}^{2}}\right) 2 \pi r d r, \\
P=\frac{c}{8 \pi} r_{0}^{2} E_{0 \mathrm{cr}}^{2}\left[\varepsilon_{0}^{\prime}(f=1)\right]^{1 / 2} .
\end{gathered}
$$

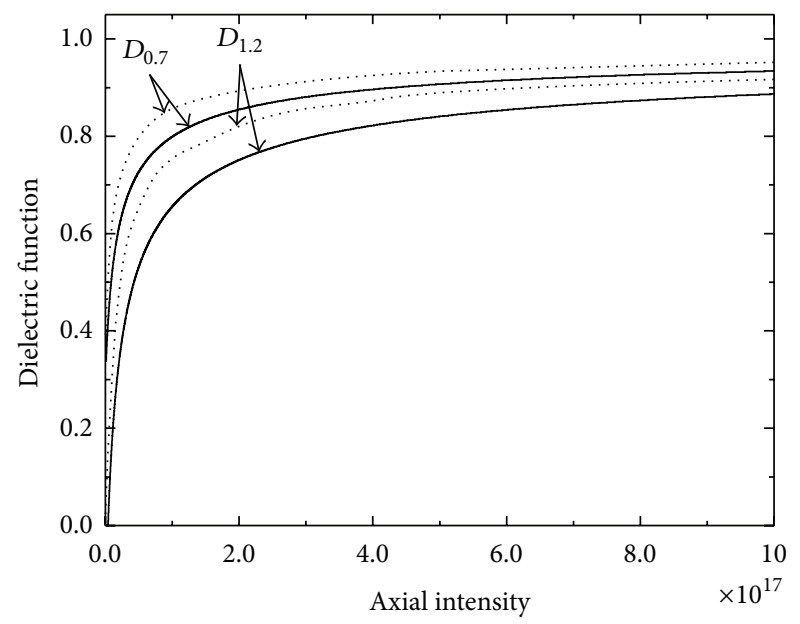

Figure 2: Variation of dielectric function $\varepsilon$ as function of axial intensity for linearly (solid line) and circularly (dot line) polarized laser beam. Curves $D_{0.7}$ and $D_{1.2}$ correspond to $\left(\omega_{p} / \omega\right)^{2}=0.7$ and 1.2 , respectively.

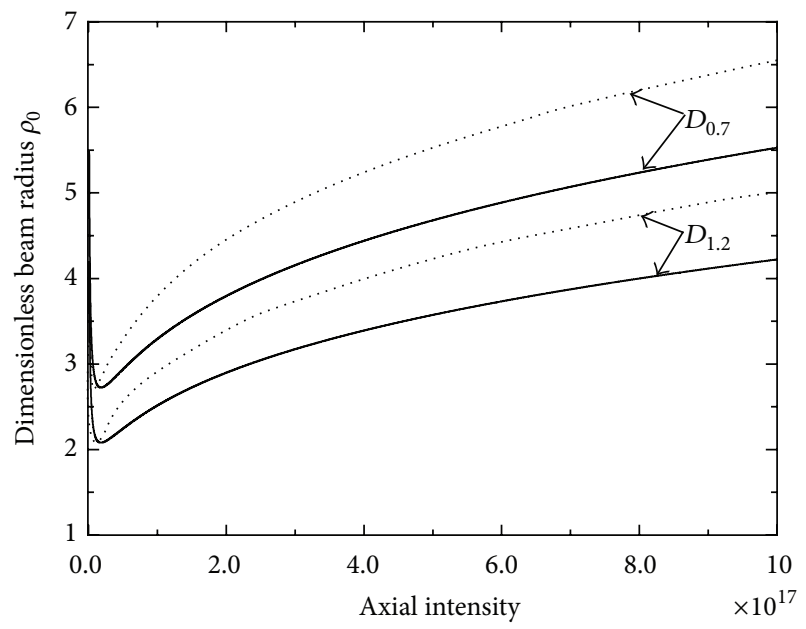

FIgURE 3: Critical curves, that is, the relation between dimensionless beam radius $\rho_{0}=\left(r_{0} \omega / c\right)$ and axial intensity for linearly (solid line) and circularly (dot line) polarized laser beam. Curves $D_{0.7}$ and $D_{1.2}$ correspond to $\left(\omega_{p} / \omega\right)^{2}=0.7$ and 1.2 , respectively.

\section{Results and Discussion}

The differential equation (39) is to study the propagation of linearly and circularly polarized beams through plasma. We have chosen parameters accessible for interaction of such beams with moderately underdense plasma to slightly overdense plasma. The numerical values are chosen for laser intensity ranging between $\mathbf{I}=10^{16}$ to $10^{18} \mathrm{~W} / \mathrm{cm}^{2}$, electron density between 0.1 to 1.5 of critical density, laser frequency $\omega=1.7 \times 10^{14} \mathrm{sec}^{-1}$ and initial radius of the beam $r_{0}$ laying between 1 to $3 \mu \mathrm{m}$. The dielectric function given by (20) and beam propagation equation (39) together with boundary conditions, are numerically solved. Further we consider the propagation of the beam in axially inhomogeneous plasma by including linear density variation in plasma. 


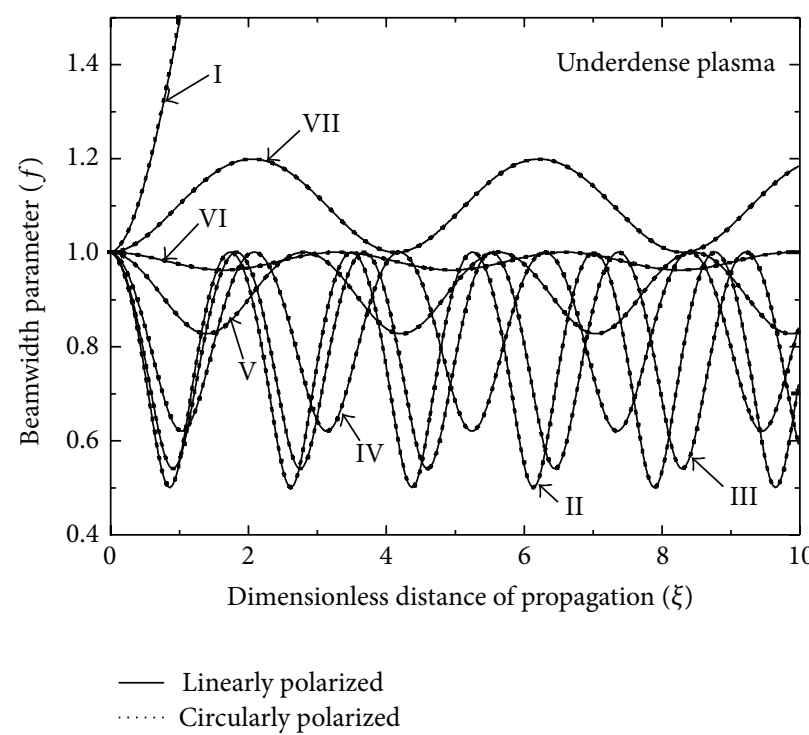

(a)

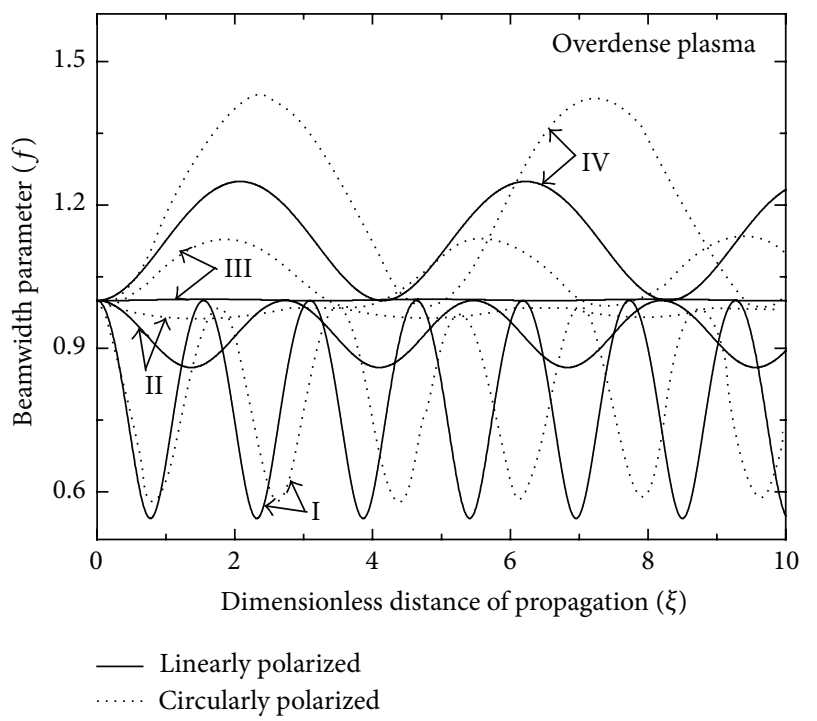

(b)

Figure 4: (a) Variation of beamwidth parameter $(f)$ with dimensionless distance of propagation $(\xi)$ for linearly (solid line) and circularly (dot line) polarized beams. Curves I, II, III, IV, V, VI, and VII correspond to $\left(P / P_{\mathrm{crl}}\right)=0.5,5,10,20,60,100$, and 200 , respectively. Here $\left(\omega_{p} / \omega\right)^{2}=0.7, \rho_{0}=4$, and $\left(P_{\mathrm{cr} 2} / P_{\mathrm{cr} 1}\right)=113.22$ (for linearly polarized laser beam) and 113.21 (for circularly polarized laser beam). (b) Variation of beamwidth parameter $(f)$ with dimensionless distance of propagation $(\xi)$ for linearly (solid line) and circularly (dot line) polarized beams. Curves I, II, III, and IV correspond to $\left(P / P_{\mathrm{cr} 1}\right)=10,60,100$, and 200, respectively. Here $\left(\omega_{p} / \omega\right)^{2}=1.2, \rho_{0}=3$, and $\left(P_{\text {cr2 }} / P_{\text {cr1 }}\right)=99.06$ (for linearly polarized laser beam) and 64.89 (for circularly polarized laser beam).

Figure 1 shows the variation of relativistic factor $\gamma$ versus intensity for linearly and circularly polarized laser beam. From the graph it is evident that the growth in relativistic factor for circularly polarized laser beam is more as compared to linearly polarized beam. Figure 2 shows plot of dielectric function versus axial intensity for linearly and circularly polarized beams in both underdense and overdense plasmas corresponding to different concentration of electron density. It is seen that initially there is an increase in dielectric function and after a certain value it attains a constant value. Because of very strong fields, the nonlinearity saturates. As a result the electromagnetic wave drives all of the plasma out of the regions of large field intensity and establishes equilibrium with radiation inside a vacuum channel surrounded by plasma. As we move from underdense region to overdense region the saturation effect slows down.

The critical curve (i.e., a plot of normalized radius of the beam " $\rho_{0}$ " against the axial intensity proportional to critical beam power) (40a) and (40b) is represented by Figure 3 for linearly and circularly polarized beams at $\Omega_{p}^{2}=\left(\omega_{p} / \omega\right)^{2}=$ 0.7 and 1.2. Equations (40a) and (40b) have two critical powers $P_{\mathrm{cr} 1}$ and $P_{\mathrm{cr} 2}$, corresponding to two different values of electric field strength (or intensity of electric field), say $E_{\text {ocr } 1}$ (or $\left.I_{\text {ocr1 }}\right)$ and $E_{\text {ocr2 } 2}$ (or $\left.I_{\text {ocr2 } 2}\right)$ such that $\left(E_{\text {ocr1 }}<E_{\text {ocr2 } 2}\right.$ ). The beam can be self-focused when its power $P$ lies between the two critical values $\left(P_{\mathrm{cr} 1}<P<P_{\mathrm{cr} 2}\right)$, and the range $\left(P_{\mathrm{cr} 2}-P_{\mathrm{cr} 1}\right)$ increases rapidly with increasing normalized radius. The region above the critical curve (i.e., $P_{\text {cr1 }}<$ $\left.P<P_{\mathrm{cr} 2}\right)$ is the self-focusing region, and region below the critical curve is known as the defocusing region. The effect of different concentration of electron density can be clearly seen from these critical curves. As concentration of electron density increases, that is, if we move from underdense region to overdense region the critical curve shifts downward.

Figures 4(a) and 4(b) show the variations of dimensionless beamwidth parameter $f$ with dimensionless distance of propagation $\xi$ at relativistic intensity in plasma for different values of electron density in underdense and overdense plasmas. From the critical curves for $\Omega_{p}^{2}=0.7\left(D_{0.7}\right)$ and $\rho_{0}=$ 4 , the value $\left(P_{\mathrm{cr} 2} / P_{\mathrm{cr} 1}\right)=113.22$ for linearly/plane polarized beam, and $\left(P_{\mathrm{cr} 2} / P_{\mathrm{cr} 1}\right)=113.21$ for circularly polarized beam. At $\Omega_{p}^{2}=1.2\left(D_{1.2}\right)$ and $\rho_{0}=3,\left(P_{\mathrm{cr} 2} / P_{\mathrm{cr} 1}\right)=99.06$ for linearly/plane polarized beam, and $\left(P_{\mathrm{cr} 2} / P_{\mathrm{cr} 1}\right)=64.889$ for circularly polarized beam. The beam shows focusing/defocusing for different values of $P / P_{\mathrm{cr} 1}$. This is because as we change the strength of the electric field, the maxima and minima get shifted. For a given value of $\rho_{0}$ when $\left(P_{\mathrm{cr} 1}<P<P_{\mathrm{cr} 2}\right)$, the second term on the RHS of (39) dominates the first term at $z=0$, and $\left(d^{2} f / d \xi^{2}\right)$ is negative; hence, $f$ decreases with the distance of propagation.

For certain value of $\xi$, the two terms on RHS of (39) get cancelled resulting in $\left(d^{2} f / d \xi^{2}\right)=0$, and for larger values of $\xi$, RHS becomes positive. However, the beam still continues to converge on account of the curvature it has already gained, though $(d f / d \xi)$ becomes less and less negative. At certain $\xi,\left(d^{2} f / d \xi^{2}\right)=0$ and $f=f_{\min }$. Beyond this point, the diffraction term dominates over the nonlinear term, and hence $f$ exhibits the oscillatory behavior. From the figures it is evident that due to relativistic mass effect, increase in effective electron mass decreases the electron plasma frequency and makes the penetration of the beam into the 


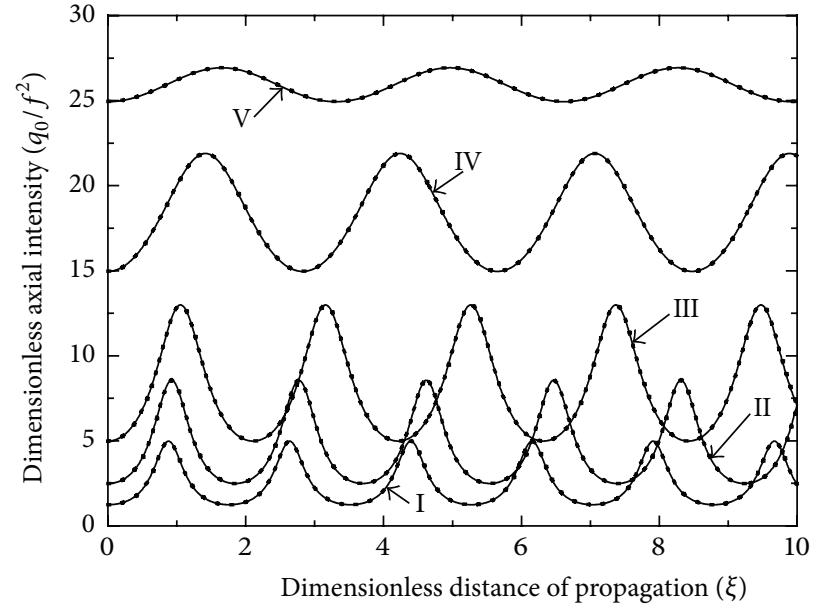

— Linearly polarized

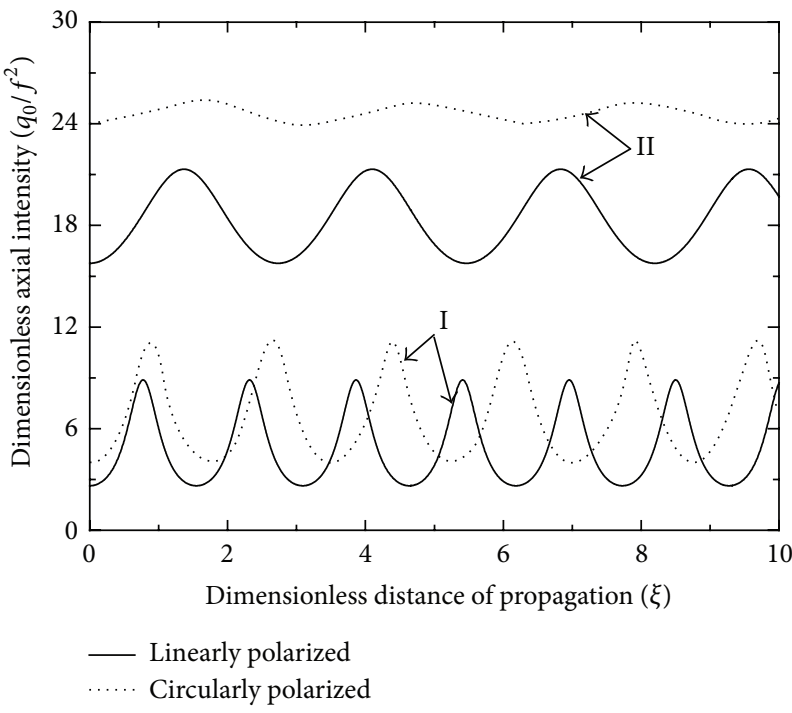

(b)

FIGURE 5: (a) Variation of dimensionless axial intensity $\left(q_{0} / f^{2}\right)$ with dimensionless distance of propagation $(\xi)$ for linearly/plane (solid line) and circularly (dot line) polarized beams. Curves I, II, III, IV, and V correspond to $\left(P / P_{\mathrm{crl}}\right)=5,10,20,60$, and 100, respectively. Remaining parameters are as mentioned in Figure 4(a). (b) Variation of dimensionless axial intensity $\left(q_{0} / f^{2}\right)$ with dimensionless distance of propagation $(\xi)$ for linearly (solid line) and circularly (dot line) polarized beams. Curves I and II to $\left(P / P_{\mathrm{crl}}\right)=10$ and 60 , respectively. Remaining parameters are as mentioned in Figure 4(b).

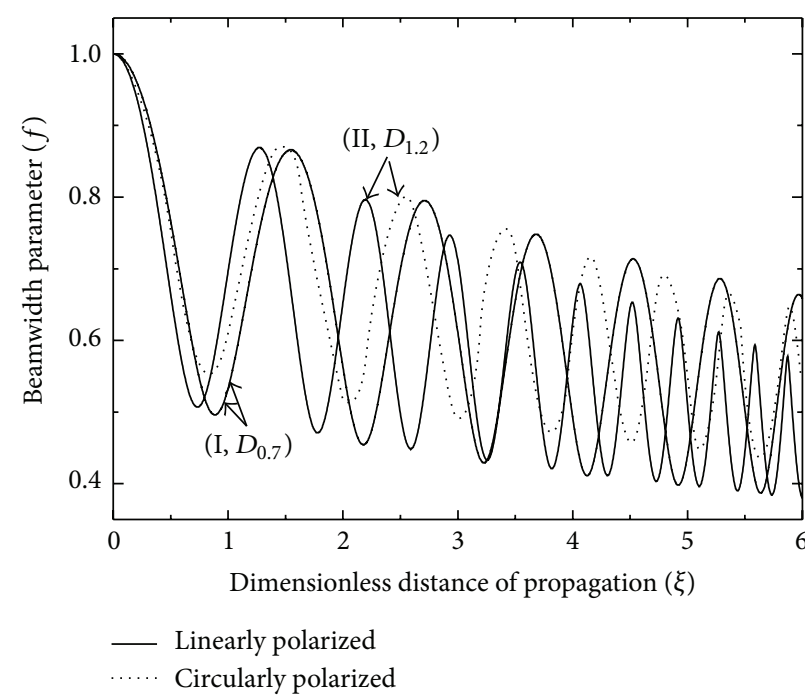

(a)

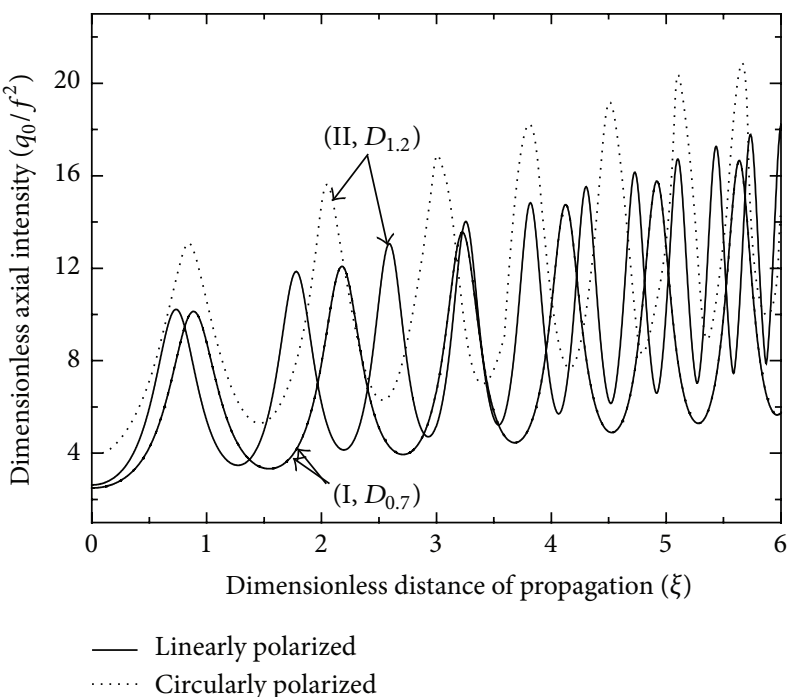

(b)

FIGURE 6: (a) Variation of beamwidth parameter $(f)$ with dimensionless distance of propagation $(\xi)$ for linearly (solid line) and circularly (dot line) polarized beams. Curves $\left(\mathrm{I}, D_{0.7}\right)$ correspond to $\left(\omega_{p} / \omega\right)^{2}=0.7(1+2.5 \xi), \rho_{0}=4$, and $\left(P_{\mathrm{cr} 2} / P_{\mathrm{cr} 1}\right)=113.22$ (for linearly polarized laser beam) and 113.21 (for circularly polarized laser beam), and curves (II, $\left.D_{1.2}\right)$ correspond to $\left(\omega_{p} / \omega\right)^{2}=1.2(1+2.5 \xi), \rho_{0}=3$, and $\left(P_{\mathrm{cr} 2} / P_{\mathrm{cr} 1}\right)=99.067$ (for linearly polarized laser beam) and 64.89 (for circularly polarized laser beam). (b) Variation of dimensionless axial intensity $\left(q_{0} / f^{2}\right)$ with dimensionless distance of propagation $(\xi)$ for linearly (solid line) and circularly (dot line) polarized beams. Curves (I, $D_{0.7}$ ) correspond to $\left(\omega_{p} / \omega\right)^{2}=0.7(1+2.5 \xi), \rho_{0}=4$, and $\left(P_{\mathrm{cr} 2} / P_{\mathrm{cr} 1}\right)=113.22$ (for linearly polarized laser beam) and 113.21 (for circularly polarized laser beam) and curves (II, $\left.D_{1.2}\right)$ correspond to $\left(\omega_{p} / \omega\right)^{2}=1.2(1+2.5 \xi), \rho_{0}=3$, and $\left(P_{\mathrm{cr} 2} / P_{\mathrm{crl}}\right)=99.067$ (for linearly polarized laser beam) and 64.89 (for circularly polarized laser beam). 
overdense region of plasma, which is known as self-induced transparency. At higher concentration of electron density beam gets more focused as compared to low concentration of electron density. The variation of dimensionless axial intensity $\left(\alpha E_{0}^{2} / f^{2}\right)=\left(q_{0} / f^{2}\right)$ of the beam with distance of propagation $\xi$ corresponding to Figures 4(a) and 4(b) for self-focusing region is depicted in Figures 5(a) and 5(b). The intensity peaks for higher concentration of electron densities are higher with a reduced distance between two successive peaks.

Figure 6(a) illustrates the variations of dimensionless beamwidth parameter $f$ with distance of propagation $\xi$ for different concentrations of electron densities in self-focusing region with linear increase in electron density. Careful observation of the results from figure indicates that for linearly increasing density plasma, minimum value of the normalized beamwidth parameter $\left(f_{\min }\right)$ for the second and higher orders decreases continuously in underdense and overdense plasmas. In underdense plasma it propagates long distance as compared to overdense plasma, but its focusing is less. The corresponding graph for variation of dimensionless axial intensity $\left(q_{0} / f^{2}\right)$ of the beam versus distance of propagation $\xi$ is shown in Figure 6(b). From the numerical analysis and graphs it is evident that both linearly polarized and circularly polarized beams can propagate through underdense plasma as well as they penetrate overdense plasma or say they exhibit self-induced transparency effect.

In underdense plasma there is not much difference in propagation of linearly polarized and circularly polarized beams, while the propagation is affected in case of overdense plasma and focal point changes.

A threshold exists for onset of self-focusing, as this effect must overcome the spreading of the beam due to diffraction. A light beam is focused to be self-trapped at any arbitrary diameter and will thus not spread. Further, self-trapping occurs at a critical power level, which is independent of the beam diameter. The theory presented here has a much wide range of application than the previous one, because it is not a perturbation treatment. Furthermore, it is applicable at all intensities-high and low.

\section{References}

[1] D. Umstadter, "Relativistic laser-plasma interactions," Journal of Physics D, vol. 36, Article ID R151, 2003.

[2] A. L. Lei, K. A. Tanaka, R. Kodama et al., "Study of ultraintense laser propagation in overdense plasmas for fast ignition," Physics of Plasmas, vol. 16, no. 5, Article ID 056307, 2009.

[3] H. Hora, "Theory of relativistic self-focusing of laser radiation in plasmas," Journal of the Optical Society of America, vol. 65, no. 8, pp. 882-886, 1975.

[4] G. Schmidt and W. Horton, "Self-focusing of laser beams in the beatwave accelerator," Comments on Plasma Physics and Controlled Fusion, vol. 9, p. 85, 1985.

[5] G. Z. Sun, E. Ott, Y. C. Lee, and P. Guzdar, "Self-focusing of short intense pulses in plasmas," Physics of Fluids, vol. 30, p. 526, 1987.

[6] M. R. Siegrist, "Self-focusing in a plasma due to ponderomotive forces and relativistic effects," Optics Communications, vol. 16, no. 3, pp. 402-407, 1976.
[7] E. Esarey, A. Ting, and P. Sprangle, "Relativistic focusing and beat wave phase velocity control in the plasma beat wave accelerator," Applied Physics Letters, vol. 53, no. 14, pp. 1266$1268,1988$.

[8] S. A. Akhmanov, A. P. Sukhorukov, and R. V. Khokhlov, "Selffocusing and diffraction of light in a nonlinear medium," Soviet Physics Uspekhi, vol. 10, no. 5, article 609, 1968.

[9] M. S. Sodha, A. K. Ghatak, and V. K. Tripathi, Self-Focusing of Laser Beams in Dielectrics, Semiconductors and Plasmas, Tata McGraw-Hill, New Delhi, India, 1974.

[10] M. Asthana, "Relativistic self-focusing of laser beams of arbitrary intensity in plasmas," Indian Journal of Pure and Applied Physics, vol. 31, p. 564, 1993.

[11] M. Asthana, M. S. Sodha, and K. P. Maheshwari, "Relativistic self-focusing of laser beams in time-harmonic plane waves: arbitrary intensity," Journal of Plasma Physics, vol. 51, no. 1, pp. $155-162,1994$. 

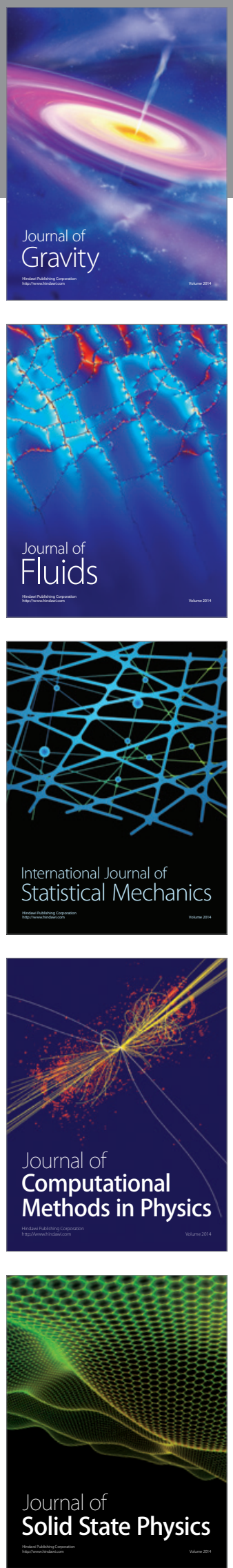

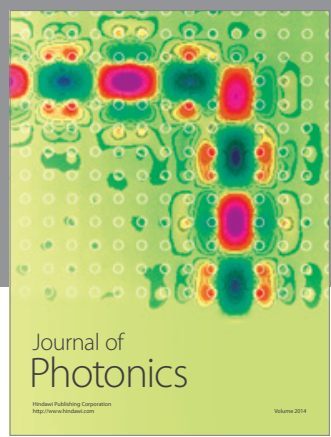

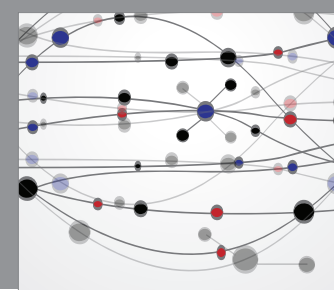

The Scientific World Journal

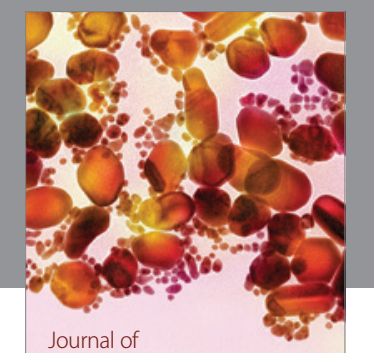

Soft Matter
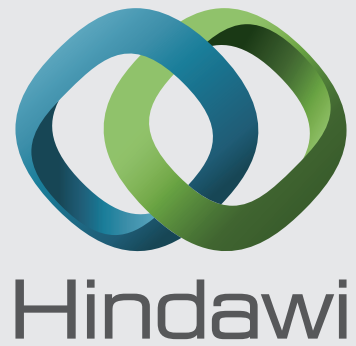

Submit your manuscripts at

http://www.hindawi.com
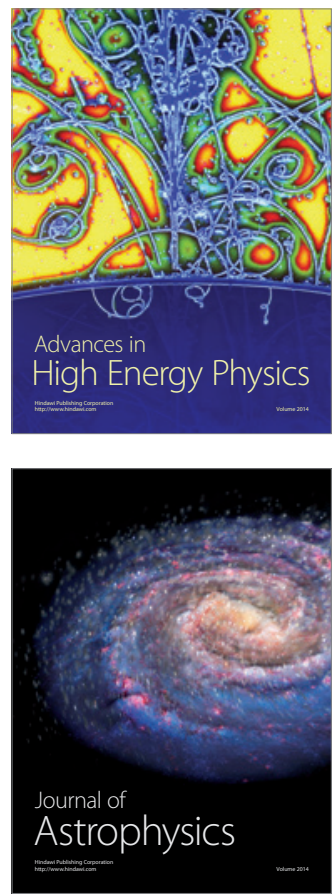
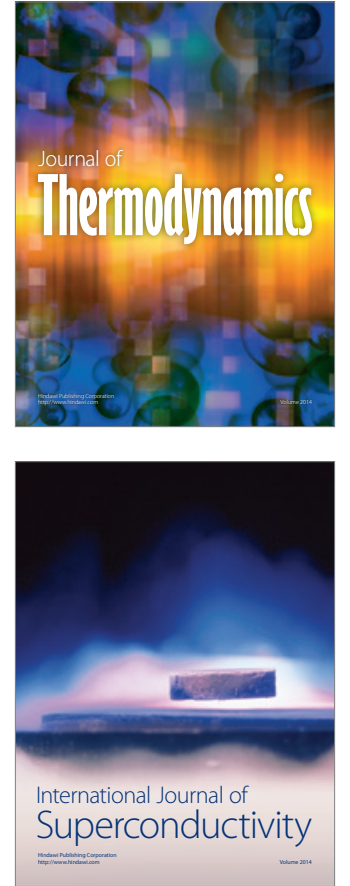
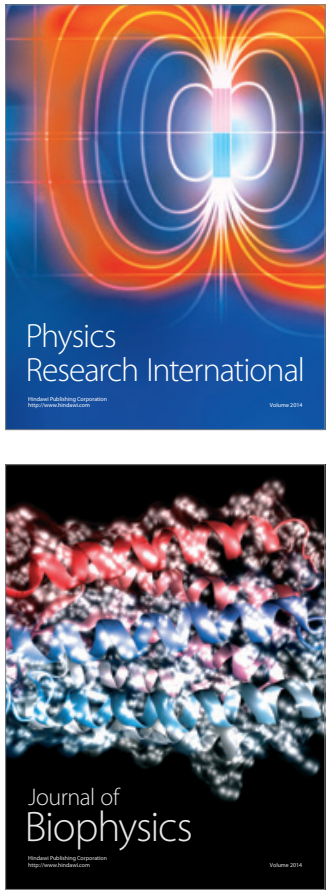
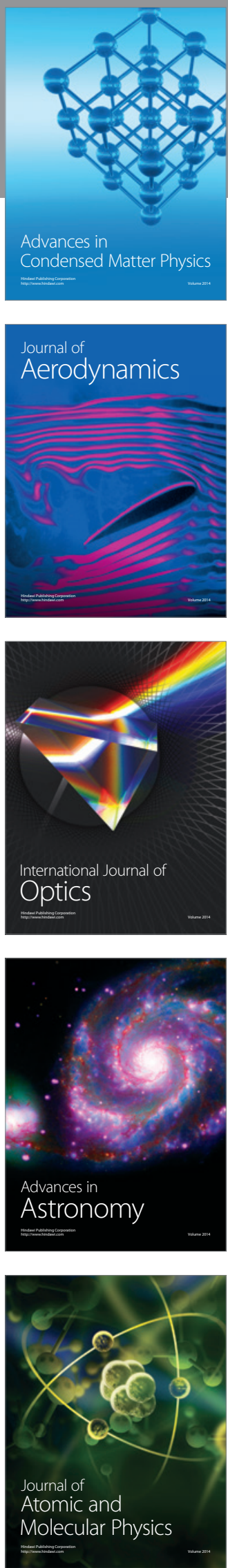